\title{
Controle de um robô móvel em um galpão de estoque utilizando Processo de Decisão de Markov Parcialmente Observável *
}

\author{
Neemias Silva Monteiro* Carlos Andrey Maia* \\ Vinicius Mariano Gonçalves* \\ * Programa de Pós-Graduação em Engenharia Elétrica - Universidade \\ Federal de Minas Gerais - Av. Antônio Carlos 6627, 31270-901, \\ Belo Horizonte, MG, Brasil (e-mail: neemias@ufmg.br, \\ $\{$ maia,mariano\}@cpdee.ufmg.br).
}

\begin{abstract}
In most robotic systems states can not be directly observed, and there is uncertainty as to the outcome of a robot's decision making. POMDP's are presented as an alternative to solve problems immersed in uncertainties, selecting actions to accomplish a given task. In this work, it was simulated a system of locating and controlling the actions of a robot that moves through a warehouse. The results of the experiments show that the POMDP presents robustness and efficiency to determine the control actions of the robot.

Resumo: Na maioria dos sistemas robóticos os estados não podem ser diretamente observados, e há incerteza quanto ao resultado da tomada de decisão de um robô. POMDP's são apresentados como alternativa para solucionar problemas imersos em incertezas, selecionando ações com objetivo de realizar uma dada tarefa. Neste trabalho, foi simulado um sistema de localização e controle das ações de um robô que se locomove por um galpão utilizado para estocagem. Os resultados dos experimentos mostram que o POMDP apresenta robustez e eficiência para determinar as ações de controle do robô.
\end{abstract}

Keywords: POMDP; Robotic; Manufacturing Systems; Decision Making; Computer Vision. Palavras-chaves: POMDP; Robótica; Sistemas de Manufatura; Tomada de Decisão; Visão Computacional.

\section{INTRODUÇÃO}

Com o avanço da tecnologia, sistemas robóticos móveis estão sendo desenvolvidos em grandes escalas e utilizados em várias aplicações. Robôs móveis podem ser classificados, com relação ao meio de locomoção, em: terrestres, aquáticos, aéreos e espaciais (Dudek and Jenkin, 2010). Estes robôs fazem uso dos mais diversos tipos de sensores para navegarem em um ambiente (Bonin-Font et al., 2008).

A robótica clássica assume que os sensores de um robô podem mensurar completamente o estado do ambiente, e que os efeitos das ações de controle são determinísticos. Entretanto, estas suposições são utópicas, pois na maioria das aplicações práticas os estados não podem ser diretamente observados, e as medições recebidas pelo robô são projeções ruidosas do estado. Além do fato que as ações executadas por um robô têm incertezas, dado que os resultados das ações são não determinísticos. Deste modo, o robô deve levar em conta as incertezas do ambiente e das suas ações quando tiver que tomar uma decisão (Thrun et al., 2005).

O MDP (Markov Decision Process) e o POMDP (Partially Observable Markov Decision Process) podem ser apresentados como alternativa para solucionar problemas que são

\footnotetext{
* Os autores agradecem as agências CNPq, CAPES, FAPEMIG, e ao Programa de Pós-Graduação em Engenharia Elétrica da UFMG, pelo apoio financeiro.
}

inerentemente carregados de incertezas (Thrun et al., 2005; Pellegrini and Wainer, 2007). Estes métodos selecionam uma sequência de ações com o objetivo de realizar uma dada tarefa, considerando as imprecisões do sistema ( $\mathrm{Pi}-$ neau et al., 2004). Diferente das cadeias de Markov, no MDP há possibilidade de um agente intervir no sistema executando ações. O MDP modela sistemas em que, os estados podem ser completamente observados sempre que uma decisão é tomada, mas cada decisão possui um resultado incerto. Para resolver um MDP deve-se encontrar a política ótima, que determina qual decisão tomar para maximizar a recompensa esperada (Pellegrini and Wainer, 2007). O POMDP é uma extensão do MDP, no qual, os estados não podem ser medidos diretamente, mas são inferidos por meio de observações indiretas (Páll et al., 2016). Por ser uma estrutura que abrange uma maior quantidade de problemas reais, e incorporar a incerteza sobre as percepções e ações, o POMDP será o objeto de estudo deste trabalho.

O POMDP é o ferramental utilizado para planejamento/controle das ações de um robô móvel terrestre, que se direciona a uma posição previamente selecionada de um galpão utilizado para estoque, em um ambiente definido topologicamente. Alguns trabalhos recentes relacionados a esta aplicação estão disponíveis em Nardi and Stachniss (2019), Wang et al. (2018) e Miehling et al. (2015). 
O artigo está organizado da seguinte forma: na Seção 2 apresenta-se a estrutura do POMDP. A Seção 3 oferece uma explanação sobre o algoritmo de iteração de valores. Na Seção 4 mostra-se uma visão geral de como o sistema foi desenvolvido. A Seção 5 exibe resultados de simulações realizadas no software V-REP. Conclusões obtidas e trabalhos futuros são relatados na Seção 6 .

\section{PROCESSO DE DECISÃO DE MARKOV PARCIALMENTE OBSERVÁVEL}

Um Processo de Decisão de Markov Parcialmente Observável (POMDP) modela processos em que: as transições entre estados são probabilísticas; interfere-se no processo executando ações; existe incerteza quanto ao resultado da ação executada; o resultado de uma ação em um estado depende apenas da ação e do estado atual do processo (propriedade Markoviana). O termo parcialmente observável, indica que as medidas são incompletas e/ou projeções ruidosas dos estados do sistema.

O POMDP pode ser definido pela tupla $P=(S, A, T, R, \Omega$, $O)$. $S$ é conjunto de estados do sistema. $A$ é o conjunto de ações que podem ser executadas. $T: S \times A \times S \longrightarrow[0,1]$ é a probabilidade do sistema passar para o estado $s^{\prime}$, dado que estava em $s$ quando a ação $a$ foi executada $\left(p\left(s^{\prime} \mid a, s\right)\right)$. $R: S \times A \longrightarrow \mathbb{R}$ é uma função que fornece a recompensa do estado $s$ quando uma ação a é executada $(R(s, a))$. A componente $\Omega$ representa um conjunto de observações que são obtidas em cada época de decisão, e $O$ é uma função que fornece a probabilidade de uma observação $o$ ser verificada, dado o estado $s(p(o \mid s))$.

No POMDP cada ação tem como resultado uma observação, que é probabilisticamente relacionada aos estados do sistema. Ao invés, de um "estado atual" do sistema, no POMDP há uma distribuição de probabilidade sobre os estados, também chamada de crença $b$ (sendo $b(s)$ a probabilidade do sistema estar no estado $s$, e $\sum_{s} b(s)=1$ ).

A crença é estabelecida a partir de um nível de confiança do estado inicial do sistema. Quando não há nenhuma informação sobre o estado, utiliza-se uma distribuição uniforme para representar $b_{0}$. Já em situações que se tem um conhecimento maior sobre o estado inicial do sistema, atribui-se um percentual $X \%$ ao mesmo, e aos demais $(1-X) /(n-1)$, no qual $n$ é o número de estados do sistema. Como o sistema é parcialmente observável, a crença $b_{k}$ deve ser atualizada a cada tomada de decisão $k-1$, utilizando as observações provenientes do sistema. Ou seja, ao executar uma ação $a_{k-1}$, percebe-se uma observação $o_{k}$, referente ao estado $s_{k}$.

A partir destes dados, e da distribuição de probabilidade anterior $b_{k-1}$, pode-se calcular uma nova crença sobre os estados:

$$
b_{k}\left(s^{\prime}\right)=p\left(o_{k} \mid s^{\prime}\right) \sum_{s \in S} p\left(s^{\prime} \mid a_{k-1}, s\right) b_{k-1}(s),
$$

o procedimento é repetido $\forall s^{\prime} \in S$. Após obter $b_{k}$ realizase a normalização, tal que $\sum_{s} b_{k}(s)=1$.

Em suma, conclui-se que no POMDP o modelo perceptivo $p(o \mid s) \mathrm{s}$, a e o modelo de transição $p\left(s^{\prime} \mid s, a\right)$ são estocásticos. A seguir, são definidos os conceitos de objetivo, política, horizonte de planejamento e função valor, termos comumente presentes em algoritmos de solução de POMDP's.

\subsection{Objetivos e Política}

Geralmente, os robôs escolhem ações que o levem até um objetivo (do inglês, goal). Os objetivos podem referir-se às configurações do espaço de trabalho. Por exemplo, para um robô móvel terrestre, um objetivo pode ser uma posição alvo $(x, y)$ e uma orientação $\phi_{z}$. Já a política $(\pi)$ é uma função que mapeia a crença $b$ em uma ação $a: \pi=b \longrightarrow a$.

\subsection{Horizonte de Planejamento}

Horizonte de planejamento é o número de épocas de decisão $(T)$ disponível para se tomar decisões. Em algumas situações basta escolher uma ação para maximizar a recompensa imediata, já em outras é apropriado escolher ações, cujo o somatório de todas as recompensas futuras seja máximo. Então, define-se o termo recompensa total esperada:

$$
C_{T}=E\left[\sum_{\tau=1}^{T} \gamma^{\tau} R\left(s_{\tau}, a_{\tau}\right)\right]
$$

O parâmetro $\gamma$ é chamado de fator de desconto, e pode ser utilizado para "dar mais importância" às primeiras $(\gamma<1)$ ou às últimas recompensas $(\gamma>1)$. O horizonte pode ser divido em três casos: $T=1, T$ finito (mas maior que um) e $T$ infinito. Para $T=1$, temos o caso "ganancioso", no qual o robô só procura minimizar a recompensa imediata. Por ser uma otimização mais simples (do que a otimização em várias etapas), pode ser aplicada para resolver problemas robóticos com baixa complexidade computacional.

\subsection{Função Valor}

A função valor mensura o valor esperado de uma política específica. Por exemplo, a política ótima para $T$ finito, encontrada de forma recursiva, sobre a crença de estados é dada por:

$$
\pi_{T}(b)=\underset{a}{\operatorname{argmax}}\left[r(b, a)+\gamma^{T} \sum_{b^{\prime}} V_{T-1}\left(b^{\prime}\right) P\left(b^{\prime} \mid a, b\right)\right]
$$

e a função valor ótima é:

$$
V_{T}(b)=\max _{a}\left[r(b, a)+\gamma^{T} \sum_{b^{\prime}} V_{T-1}\left(b^{\prime}\right) P\left(b^{\prime} \mid a, b\right)\right] .
$$

Mais detalhes sobre o POMDP podem ser encontrados em Pellegrini and Wainer (2007) e Thrun et al. (2005).

\section{ITERAÇÃO DE VALORES}

A abordagem mais difundida para calcular a política $\pi$ de sistemas probabilísticos, incluindo o POMDP, é o algoritmo de iteração de valores (Howard, 1960; Bellman, 1957). Este algoritmo utiliza programação dinâmica para calcular a função valor ótima $V^{*}(b)$ em cada época de decisão. Para tal, calcula-se a utilidade de cada ação em relação à função de recompensa.

Neste trabalho utiliza-se mundos finitos, em que o espaço de observações, ações e estados são finitos. Quando estas condições são obedecidas, o algoritmo de iteração de valores fornece uma solução exata para o problema de calcular a função valor ótima. Observando (3) e (4), nota-se que 
o somatório é sobre todas as crenças $b$. Se no sistema há $n$ estados (finitos), o espaço de todas as crenças é contínuo e de dimensão $n-1$ (quando se conhece $b\left(s_{1}\right)$ a $b\left(s_{n-1}\right)$ pode-se especificar $b(s)$, usando o somatório $\left.\sum_{s} b(s)=1\right)$. Assim, realizar o somatório sobre o espaço de crenças demanda alta complexidade computacional. Dada a dificuldade natural do algoritmo de iteração de valores, a mesma é amplificada quando se utiliza horizonte de planejamento $T>1$.

Devido às dificuldades mencionadas acima, no presente trabalho é realizada uma adaptação no algoritmo de iteração de valores apresentado em Thrun et al. (2005). Ao invés de usar $T$ finito maior que um, foram realizados ajustes para se utilizar $T=1$. Portanto, a partir das modificações realizadas, diminui-se a complexidade computacional (a fim do algoritmo ser implementado computacionalmente com eficiência) e maximiza-se a recompensa imediata. $\mathrm{O}$ resultado das adaptações no algoritmo de iteração de valores de Thrun et al. (2005) pode ser visto no Algoritmo 1 (o Algoritmo 1 resolve (3) e (4) para $T=1$ ).

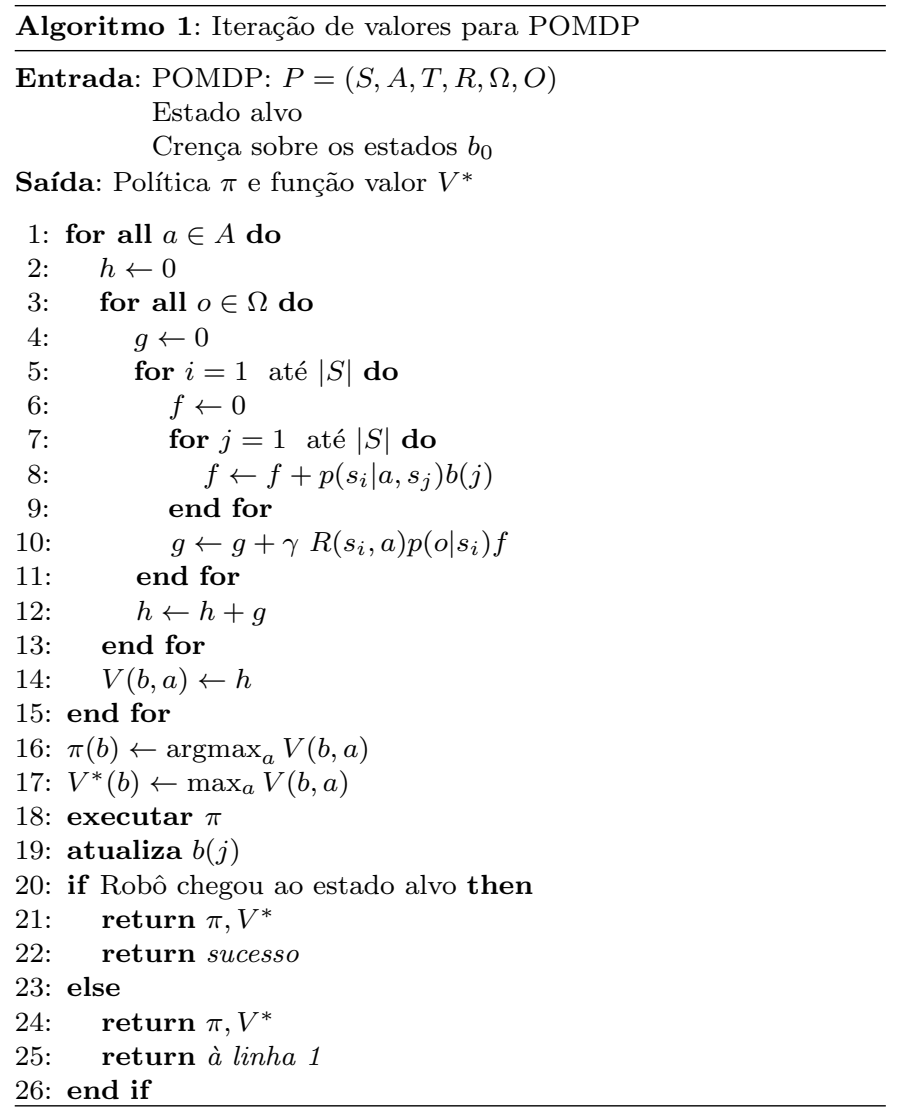

O Algoritmo 1 recebe como entrada o POMDP $P$, o estado marcado como alvo, e a crença inicial sobre os estados. Ele fornece como saída a política $\pi(b)$ a ser executada e a função valor $V^{*}(b)$ associada à ela. Das linhas 6 à 9 , verifica-se a possibilidade do sistema passar para o estado $s_{i}$, das linhas 4 à 11 calcula-se a probabilidade de se observar $o$ no estado $s_{i}$ e a recompensa do estado $s_{i}$, quando a ação $a$ for executada. Este processo é repetido $\forall s_{i} \in S$. Entre as linhas 1 à 15 realiza-se o processo acima $\forall a \in A$ e $\forall o \in \Omega$. Nas linhas 16 e 17 computa-se a política $\pi$ que maximiza a recompensa imediata da função valor $V^{*}$. Após implementar a política $\pi$ (linha 18), o robô deve atualizar a crença de estados $b(j)$ (linha 19) utilizando (1).
Caso b(estado_alvo $)>0.8$, considera-se que o robô chegou ao alvo (linha 20), e o algoritmo encerra-se retornando sucesso (linha 22). Se o estado alcançado não for o alvo $($ b(estado_alvo $) \leq 0.8)$, retorna-se à linha 1 e repete o processo descrito acima.

A recompensa $R\left(s_{i}, a\right)$ também pode ser escrita por $R\left(a_{k, i} \mid s_{k}\right)$, simbolizando a recompensa de se executar a ação que leva o robô do estado $s_{k}$ para o $s_{i}\left(a_{k, i}\right)$, dado que o robô está no estado $s_{k} . R\left(a_{k, i} \mid s_{k}\right)$ é definida por:

$$
R\left(a_{k, i} \mid s_{k}\right)=\frac{1}{\left(D_{k}-D_{i}-d_{k i}\right)^{2}+1} .
$$

$D_{k}$ e $D_{i}$ representam as distâncias ótimas dos estados $s_{k}$ e $s_{i}$ ao estado alvo, respectivamente, e $d_{k i}$ a distância entre o estado $s_{k}$ e $s_{i}$. $D_{k}-D_{i}-d_{k i} \leq 0 \forall i, k$. Se o caminho entre os estados $k$ e $i$ é ótimo $D_{k}-D_{i}-d_{k i}=0$, caso contrário, $D_{k}-D_{i}-d_{k i}<0$. Desta forma, $R\left(a_{k, i} \mid s_{k}\right)=1$ quando $a_{k, i}$ pertence ao caminho ótimo, e $R\left(a_{k, i} \mid s_{k}\right)$ se aproxima de zero nos casos que $a_{k, i}$ não pertence ao caminho ótimo. As distâncias ótimas $D_{k}$ e $D_{i}$ são obtidas por meio da implementação do algoritmo de Dijkstra. O algoritmo de Dijkstra calcula o caminho ótimo de um estado "alvo" aos demais estados de um grafo, de acordo com algum critério de otimalidade, por exemplo: distância euclidiana e tempo gasto no trajeto. Quando é utilizado o tempo gasto no percurso, $D_{k}$ e $D_{i}$ são substituídas por $\top_{k}$ e $\top_{i}$, ou seja, o tempo de percurso ótimo de $s_{k}$ e $s_{i}$ ao estado alvo, e $d_{k i}$ é substituída por $t_{k i}$, tempo gasto do estado $s_{k}$ ao $s_{i}$. Equação (5) é válida somente quando há conexão factível entre os estados $s_{k}$ e $s_{i}$, de outro modo, $R\left(a_{k, i} \mid s_{k}\right)=0$.

\section{VISÃO GERAL DO SISTEMA}

Neste trabalho, foi simulado um sistema de controle das ações de um robô móvel terrestre, em um ambiente definido topologicamente. O objetivo do sistema desenvolvido é estimar em qual estado do ambiente o robô está localizado, e a partir da estimativa selecionar uma ação de controle que o direcione a um estado marcado como alvo. Para tal, fazse uso do Algoritmo 1, que retorna ações com recompensas imediatas maximizadas. Uma perspectiva do sistema é mostrada na Figura 1. O sistema representa um galpão aberto utilizado para estoque de oito pilhas de produtos (os produtos podem ser dos mais diversos tipos, e as pilhas são simbolizadas com os blocos cinza). O ambiente simulado possui $17 \times 13 \mathrm{~m}$ de dimensão, e um robô Pioneer $3-D X$ se locomove por ele. Durante a movimentação, o robô observa cilindros coloridos, que são usados para estimar a posição do mesmo. O Pioneer 3-DX é equipado com uma câmera kinect e um sensor de distância laser SICK.

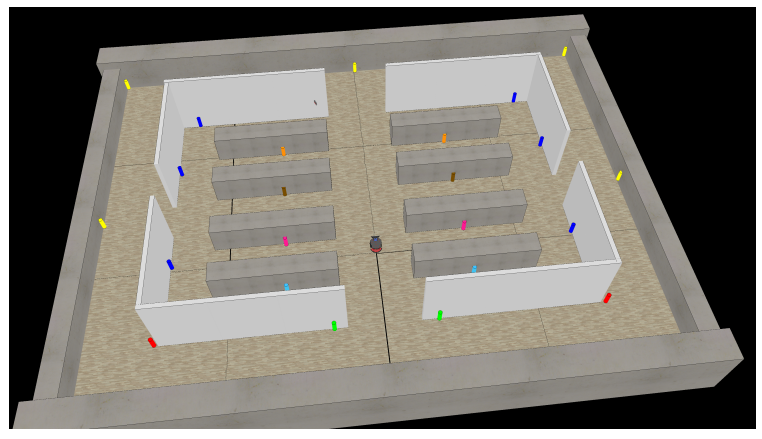

Figura 1. Sistema de estocagem proposto. 


\section{$4.1 V$-REP e ROS}

O sistema de estocagem foi construído no simulador $\mathrm{V}$ REP, um ambiente baseado em arquitetura de controle distribuído, no qual cada objeto pode ser controlado individualmente por um script interno (em linguagem LUA) ou externo (um nó do ROS (Robot Operating System), por exemplo). Neste trabalho, utilizou-se o ROS para integrar o sistema. Foram criados dois nós, um é o script em $\mathrm{C}++$ e o outro é o próprio V-REP (usando a aplicação $V$-REP RosPlugin). Para estabelecer a comunicação entre estes nós foram gerados seis tópicos (Vel_linear, Vel_angular, Kinect_ang, Imagem_rgb, Laser, Odometria), que podem ser visualizados na Figura 2.

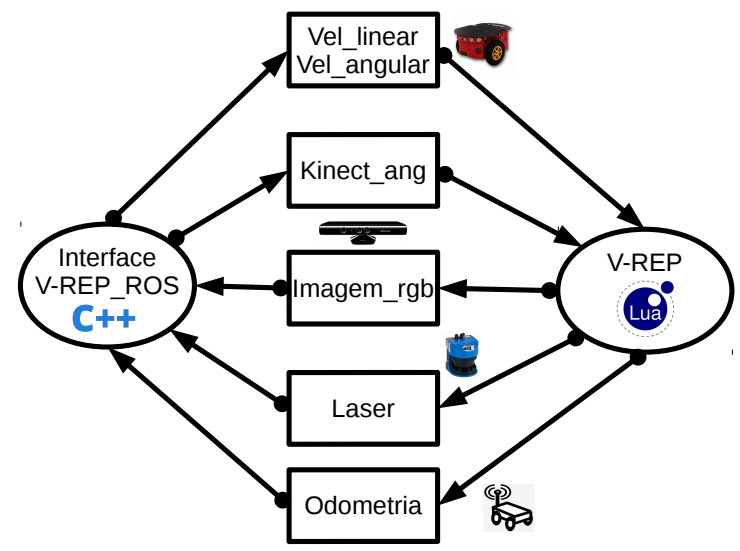

Figura 2. Integração do sistema utilizando o ROS.

No script em $\mathrm{C}++$ comanda-se o sistema, realizando o processamento das imagens provenientes do V-REP e enviando os comandos de velocidades para o robô (após realizar o planejamento de movimento). O V-REP simbolizando o "mundo real" envia as medições do laser (para evitar colisões) e de odometria para o script em $\mathrm{C}++$. Como o kinect tem um range de $57^{\circ}$ de cobertura, é necessário que sejam enviados ângulos de giro para o kinect, a fim de cobrir todo o espaço ao redor do robô.

\subsection{Mapa Topológico}

A representação topológica consiste em reproduzir o espaço de trabalho em grafos formados por estados (nós) e arestas. Os estados podem representar locais de interesse no mapa, e as arestas a conexão entre estes locais. Uma abstração topológica do sistema é exibida na Figura 3.

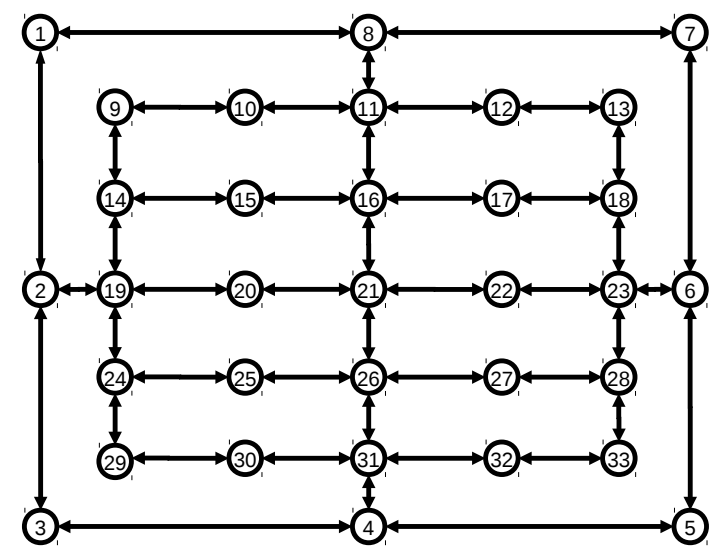

Figura 3. Mapa topológico do sistema proposto.
O mapa topológico é construído a priori e armazenado em um banco de dados. A partir destes dados, são determinadas as posições dos 33 estados no ambiente, e as conexões entre eles (e consequentemente as 88 ações factíveis). Os estados e as arestas que os conectam estão em configurações livre do ambiente. Os estados também são associados com as suas respectivas percepções, como mostra a Tabela 1.

Ao todo, há 11 tipos de observações possíveis ( $o_{1}$ a $\left.o_{11}\right)$, compostas de cilindros de oito cores: amarela, azul, ciano, laranja, marrom, rosa, vermelha e verde. Por exemplo, a observação $o_{1}$ é constituída de um cilindro amarelo e um vermelho, e pode ser vista nos estados 1, 3, 5 e 7 .

Uma particularidade do sistema é a sua simetria. Isto se torna uma dificuldade, pois há muita ambiguidade ao se relacionar uma observação a um estado. Como no exemplo mostrado acima, quando o robô detecta um cilindro amarelo e um vermelho, ele não consegue distinguir se está no estado 1, 3, 5 ou 7. Se a estimação da localização estiver incorreta, a seleção de uma ação pode resultar em colisão com o ambiente.

\subsection{Processamento de Imagem}

A aquisição da imagem é feita utilizando o sensor kinect. Para identificar os cilindros presentes na imagem, utilizase o processamento de imagem. A imagem é capturada no modelo de cores RGB (Red, Green, Blue). Esse modelo é fundamentado nas três cores primárias: vermelho, verde e azul. A partir das componentes RGB, realiza-se a conversão ${ }^{1}$ para um novo modelo de cores denominado HSV (Hue, Saturation, Value).

Na representação HSV cada cor possui uma: intensidade de brilho (Value), saturação (Saturation) que é representada pela quantidade de luz branca presente na cor, e a matiz (Hue) que é a tonalidade da cor predominante. Estes três parâmetros são calibrados para identificar as oito cores utilizadas nos cilindros.

Após aplicar o filtro de cores HSV, utiliza-se o filtro canny para realçar as componentes da imagem, e na sequência utiliza-se o comando findcontours para encontrar contornos na imagem. O processamento de imagem foi desenvolvido utilizando a biblioteca OpenCV integrada ao ROS, e retorna a quantidade de cilindros (de cada cor) presentes na imagem capturada.

\subsection{Planejamento de Movimento}

Quando uma ação $a_{k, i}$ é selecionada pelo algoritmo de iteração de valores, a mesma deve ser executada em um tempo $\tau$, no qual $\tau=d_{k i} / v_{m e d}\left(v_{m e d}\right.$ é a velocidade média entre $s_{k}$ e $s_{i}$ ). É desejado que o robô saia de $s_{k}$ e chegue em $s_{i}$ com velocidade nula. Desta forma, estabeleceu-se uma trajetória parametrizada pelo tempo, que conecta $s_{k}$ e $s_{i}$, sujeita às restrições de posição e velocidade mencionadas acima:

$$
\left[\begin{array}{l}
x_{t} \\
y_{t}
\end{array}\right]=\left[\begin{array}{l}
x_{k} \\
y_{k}
\end{array}\right]+\left[\begin{array}{l}
x_{i}-x_{k} \\
y_{i}-y_{k}
\end{array}\right] \cdot\left(\frac{-2 t^{3}}{\tau^{3}}+\frac{3 t^{2}}{\tau^{2}}\right) .
$$

1 As fórmulas para conversão do modelo de cores RGB para HSV podem ser encontradas em Chernov et al. (2015). 
Tabela 1. Observações referentes à cada estado.

\begin{tabular}{|c||c|c|c|c|c|c|c|c|c|c|c|}
\hline $\begin{array}{c}\text { Observações } \\
\text { (estados) }\end{array}$ & $\begin{array}{c}o_{1} \\
(1,3,\end{array}$ & $\begin{array}{c}O_{2} \\
(2,4,\end{array}$ & $\begin{array}{c}o_{3} \\
(9,13,\end{array}$ & $\begin{array}{c}o_{4} \\
(10,12,\end{array}$ & $\begin{array}{c}O_{5} \\
(11,31)\end{array}$ & $\begin{array}{c}o_{6} \\
(14,18,\end{array}$ & $\begin{array}{c}o_{7} \\
(15,17,\end{array}$ & $\begin{array}{c}o_{8} \\
(16,26)\end{array}$ & $\begin{array}{c}o_{9} \\
(19,23)\end{array}$ & $\begin{array}{c}o_{10} \\
(20,22)\end{array}$ & $\begin{array}{c}o_{11} \\
(21)\end{array}$ \\
\hline \hline Amarela & 1 & 1 & 0 & 0 & 1 & 0 & 0 & 0 & 1 & 0 & 0 \\
\hline Azul & 0 & 0 & 2 & 1 & 0 & 2 & 0 & 0 & 2 & 0 & 0 \\
\hline Ciano & 0 & 0 & 1 & 1 & 2 & 0 & 0 & 0 & 0 & 0 & 0 \\
\hline Laranja & 0 & 0 & 0 & 0 & 0 & 1 & 1 & 2 & 0 & 0 & 0 \\
\hline Marrom & 0 & 0 & 0 & 0 & 0 & 0 & 0 & 0 & 2 & 2 & 4 \\
\hline Rosa & 0 & 0 & 0 & 0 & 0 & 1 & 1 & 2 & 0 & 0 & 0 \\
\hline Vermelha & 1 & 0 & 0 & 0 & 0 & 0 & 0 & 0 & 0 & 0 & 0 \\
\hline Verde & 0 & 1 & 0 & 0 & 0 & 0 & 0 & 0 & 0 & 0 & 0 \\
\hline
\end{tabular}

Em (6) descreve-se o rastreamento da posição do robô durante a navegação entre $s_{k}$ e $s_{i}$, para cada $t \in[0, \tau]$. O par $\left(x_{t}, y_{t}\right)$ é a referência a ser seguida por um controlador feedback linearization ${ }^{2}$. O controlador fornece as velocidades $(v, \omega)$ que serão aplicadas ao Pioneer 3-DX:

$$
\begin{gathered}
{\left[\begin{array}{c}
\dot{x}_{r} \\
\dot{y}_{r}
\end{array}\right]=\left[\begin{array}{c}
\dot{x}_{t} \\
\dot{y}_{t}
\end{array}\right]+k_{p} \cdot\left[\begin{array}{l}
x_{t}-x_{r} \\
y_{t}-y_{r}
\end{array}\right],} \\
{\left[\begin{array}{c}
v \\
\omega
\end{array}\right]=\left[\begin{array}{cc}
\cos \left(\theta_{r}\right) & \operatorname{sen}\left(\theta_{r}\right) \\
-\operatorname{sen}\left(\theta_{r}\right) / d & \cos \left(\theta_{r}\right) / d
\end{array}\right] \cdot\left[\begin{array}{c}
\dot{x}_{r} \\
\dot{y}_{r}
\end{array}\right] .}
\end{gathered}
$$

O ganho do controlador é representado por $k_{p}$ e $\left(x_{r}, y_{r}, \theta_{r}\right)$ é a informação obtida pelo "ground truth" do V-REP. O parâmetro $d$ é um ponto próximo ao centro do robô, e é usado para evitar singularidades. Para determinar $p\left(s^{\prime} \mid s, a\right)$ fez-se uso do modelo probabilístico de movimento baseado em odometria, disponível em Thrun et al. (2005).

\section{RESULTADOS E DISCUSSÕES}

Nesta seção, mostra-se os resultados obtidos a partir de experimentos com o sistema de estocagem simulado no V-REP (aplicando o Algoritmo 1). Foi considerado que entre os estados "externos" (1-2-3-4-5-6-7-8) a velocidade de movimentação do robô é $50 \%$ maior que entre os estados "internos" (demais). Esta premissa foi estipulada, pois há maior distância entre os estados "externos", e o caminho entre eles possui menos obstrução. A seguir, são mostrados três testes, em que se construiu $R\left(a_{k, i} \mid s_{k}\right)$ considerando a distância euclidiana $\left(D_{k}, D_{i}\right.$ e $\left.d_{k i}\right)$, e também o tempo $\left(\top_{k}, \top_{i}\right.$ e $\left.t_{k i}\right)$ gasto pelo robô no trajeto entre os estados. Vídeos ilustrando os testes estão disponíveis em: https://www.youtube.com/playlist?list= PLk8QJI104X0Bw-3X7dQ57KULDAH61iX3o.

\subsection{Teste 1}

Para o primeiro teste, considerou-se que havia uma confiança de $40 \%$ do robô estar no estado $s_{3}$, e que o mesmo tinha como alvo o estado $s_{13}$. O resultado da seleção de ações com maior função valor $V(b, a)$, para o critério de menor distância percorrida, em cada época de decisão $k$, é mostrado na Tabela 2. A Tabela 2 apresenta os três maiores valores de $V(b, a)$ em cada época de decisão. Como em $k=1$, a ação $a_{3,2}$ (ação que leva o robô do estado $s_{3}$ para o $s_{2}$ ) apresenta maior função valor, a mesma é executada e atualiza-se a crença sobre os estados utilizando (1). O processo é repetido para demais épocas de decisão, até que

2 Mais informações sobre o feedback linearization são apresentadas no livro "Robot modeling and control" de Spong et al. (2006). o critério de parada do Algoritmo 1 (b(estado_alvo) > 0.8) seja atingido.

Tabela 2. Ações selecionadas entre os estados $s_{3}$ e $s_{13}$, para o critério de menor distância.

\begin{tabular}{|c||c|c|c|}
\hline$k$ & $a_{i, j} \rightarrow V(b, a)$ & $a_{i, j} \rightarrow V(b, a)$ & $a_{i, j} \rightarrow V(b, a)$ \\
\hline \hline 1 & $a_{3,2} \rightarrow 0.12$ & $a_{6,23} \rightarrow 0.09$ & $a_{3,4} \rightarrow 0.07$ \\
\hline 2 & $a_{2,19} \rightarrow 0.75$ & $a_{6,23} \rightarrow 0.17$ & $a_{8,11} \rightarrow 0.05$ \\
\hline 3 & $a_{19,14} \rightarrow 0.47$ & $a_{19,20} \rightarrow 0.40$ & $a_{19,2} \rightarrow 0.08$ \\
\hline 4 & $a_{14,9} \rightarrow 0.64$ & $a_{14,15} \rightarrow 0.26$ & $a_{14,19} \rightarrow 0.07$ \\
\hline 5 & $a_{9,10} \rightarrow 0.82$ & $a_{9,14} \rightarrow 0.12$ & $a_{14,15} \rightarrow 0.04$ \\
\hline 6 & $a_{10,11} \rightarrow 0.89$ & $a_{15,16} \rightarrow 0.06$ & $a_{10,9} \rightarrow 0.05$ \\
\hline 7 & $a_{11,12} \rightarrow 0.59$ & $a_{11,16} \rightarrow 0.19$ & $a_{11,8} \rightarrow 0.12$ \\
\hline 8 & $a_{12,13} \rightarrow 0.79$ & $a_{12,11} \rightarrow 0.14$ & $a_{17,18} \rightarrow 0.05$ \\
\hline
\end{tabular}

Com as mesmas configurações acima, porém usando o tempo como critério de otimalidade, constrói-se a Tabela 3. Comparando a Tabela 2 e a Tabela 3, observa-se que as ações para chegar em $s_{13}$, partindo de $s_{3}$ não são as mesmas. Confirmando assim, que critérios de otimalidade diferentes podem resultar em diferentes caminhos entre dois estados.

Tabela 3. Ações selecionadas entre os estados $s_{3}$ e $s_{13}$, para o critério de menor tempo.

\begin{tabular}{|c||c|c|c|}
\hline$k$ & $a_{i, j} \rightarrow V(b, a)$ & $a_{i, j} \rightarrow V(b, a)$ & $a_{i, j} \rightarrow V(b, a)$ \\
\hline \hline 1 & $a_{3,2} \rightarrow 0.11$ & $a_{6,23} \rightarrow 0.09$ & $a_{3,4} \rightarrow 0.07$ \\
\hline 2 & $a_{2,1} \rightarrow 0.46$ & $a_{6,23} \rightarrow 0.32$ & $a_{2,19} \rightarrow 0.10$ \\
\hline 3 & $a_{1,8} \rightarrow 0.89$ & $a_{7,6} \rightarrow 0.08$ & $a_{1,2} \rightarrow 0.02$ \\
\hline 4 & $a_{8,11} \rightarrow 0.97$ & $a_{11,12} \rightarrow 0.02$ & $a_{8,1} \rightarrow 0.01$ \\
\hline 5 & $a_{11,12} \rightarrow 0.59$ & $a_{11,16} \rightarrow 0.18$ & $a_{11,8} \rightarrow 0.12$ \\
\hline 6 & $a_{12,13} \rightarrow 0.79$ & $a_{12,11} \rightarrow 0.14$ & $a_{17,18} \rightarrow 0.06$ \\
\hline
\end{tabular}

\subsection{Teste 2}

O Teste 1 foi repetido, entretanto, considerou-se uma confiança de $20 \%$ do robô estar inicialmente no estado $s_{11}$, e o objetivo final era alcançar o estado $s_{5}$. O resultado para o critério de menor distância é mostrado na Tabela 4 e o de menor tempo na Tabela 5.

Tabela 4. Ações selecionadas entre os estados $s_{11}$ e $s_{5}$, para o critério de menor distância.

\begin{tabular}{|c||c|c|c|}
\hline$k$ & $a_{i, j} \rightarrow V(b, a)$ & $a_{i, j} \rightarrow V(b, a)$ & $a_{i, j} \rightarrow V(b, a)$ \\
\hline \hline 1 & $a_{11,16} \rightarrow 0.25$ & $a_{16,21} \rightarrow 0.06$ & $a_{11,12} \rightarrow 0.05$ \\
\hline 2 & $a_{16,21} \rightarrow 0.82$ & $a_{16,17} \rightarrow 0.08$ & $a_{26,31} \rightarrow 0.04$ \\
\hline 3 & $a_{21,26} \rightarrow 0.65$ & $a_{21,22} \rightarrow 0.29$ & $a_{21,16} \rightarrow 0.04$ \\
\hline 4 & $a_{26,31} \rightarrow 0.88$ & $a_{26,21} \rightarrow 0.09$ & $a_{26,25} \rightarrow 0.01$ \\
\hline 5 & $a_{31,4} \rightarrow 0.76$ & $a_{31,26} \rightarrow 0.11$ & $a_{4,5} \rightarrow 0.10$ \\
\hline 6 & $a_{4,5} \rightarrow 0.63$ & $a_{4,31} \rightarrow 0.35$ & $a_{4,3} \rightarrow 0.01$ \\
\hline
\end{tabular}


Tabela 5. Ações selecionadas entre os estados $s_{11}$ e $s_{5}$, para o critério de menor tempo.

\begin{tabular}{|c||c|c|c|}
\hline$k$ & $a_{i, j} \rightarrow V(b, a)$ & $a_{i, j} \rightarrow V(b, a)$ & $a_{i, j} \rightarrow V(b, a)$ \\
\hline \hline 1 & $a_{11,8} \rightarrow 0.17$ & $a_{16,21} \rightarrow 0.07$ & $a_{19,2} \rightarrow 0.05$ \\
\hline 2 & $a_{8,7} \rightarrow 0.50$ & $a_{8,11} \rightarrow 0.38$ & $a_{11,12} \rightarrow 0.10$ \\
\hline 3 & $a_{7,6} \rightarrow 0.99$ & $a_{7,8} \rightarrow 0.01$ & $a_{17,18} \rightarrow 0.00$ \\
\hline 4 & $a_{6,5} \rightarrow 0.85$ & $a_{6,23} \rightarrow 0.10$ & $a_{6,7} \rightarrow 0.05$ \\
\hline
\end{tabular}

\subsection{Teste 3}

O terceiro teste é semelhantes aos anteriores, porém, o robô sai de um estado, alcança um estado alvo, e depois retorna ao estado inicial. O estado inicial é o $s_{6}$ (com $30 \%$ de confiança) e o final é o $s_{29}$. Quando chegar ao estado final $\left(s_{29}\right)$, o robô deve recalcular $R\left(a_{k, i} \mid s_{k}\right)$ tomando como referência o "novo estado alvo" $\left(s_{6}\right)$. O resultado desta simulação é mostrado na Tabela 6 .

Tabela 6. Ações selecionadas entre os estados $s_{6} \rightarrow s_{29} \rightarrow s_{6}$, para o critério de menor distância.

\begin{tabular}{|c||c|c|c|}
\hline$k$ & $a_{i, j} \rightarrow V(b, a)$ & $a_{i, j} \rightarrow V(b, a)$ & $a_{i, j} \rightarrow V(b, a)$ \\
\hline \hline 1 & $a_{6,23} \rightarrow 0.50$ & $a_{22,21} \rightarrow 0.05$ & $a_{16,21} \rightarrow 0.03$ \\
\hline 2 & $a_{23,22} \rightarrow 0.71$ & $a_{23,28} \rightarrow 0.24$ & $a_{7,8} \rightarrow 0.01$ \\
\hline 3 & $a_{22,21} \rightarrow 0.99$ & $a_{22,23} \rightarrow 0.00$ & $a_{17,16} \rightarrow 0.00$ \\
\hline 4 & $a_{21,20} \rightarrow 0.58$ & $a_{21,26} \rightarrow 0.38$ & $a_{21,16} \rightarrow 0.02$ \\
\hline 5 & $a_{20,19} \rightarrow 0.98$ & $a_{20,21} \rightarrow 0.01$ & $a_{25,26} \rightarrow 0.00$ \\
\hline 6 & $a_{19,24} \rightarrow 0.77$ & $a_{19,2} \rightarrow 0.15$ & $a_{19,14} \rightarrow 0.05$ \\
\hline 7 & $a_{24,29} \rightarrow 0.89$ & $a_{24,19} \rightarrow 0.09$ & $a_{24,25} \rightarrow 0.01$ \\
\hline 8 & $a_{29,24} \rightarrow 0.70$ & $a_{29,30} \rightarrow 0.30$ & $a_{3,4} \rightarrow 0.00$ \\
\hline 9 & $a_{24,19} \rightarrow 0.78$ & $a_{24,25} \rightarrow 0.17$ & $a_{24,29} \rightarrow 0.03$ \\
\hline 10 & $a_{19,20} \rightarrow 0.72$ & $a_{19,2} \rightarrow 0.15$ & $a_{19,14} \rightarrow 0.05$ \\
\hline 11 & $a_{20,21} \rightarrow 0.94$ & $a_{20,19} \rightarrow 0.04$ & $a_{25,26} \rightarrow 0.01$ \\
\hline 12 & $a_{21,22} \rightarrow 0.73$ & $a_{21,16} \rightarrow 0.10$ & $a_{21,26} \rightarrow 0.09$ \\
\hline 13 & $a_{22,23} \rightarrow 0.81$ & $a_{22,21} \rightarrow 0.14$ & $a_{4,5} \rightarrow 0.03$ \\
\hline 14 & $a_{23,6} \rightarrow 0.75$ & $a_{23,22} \rightarrow 0.09$ & $a_{23,18} \rightarrow 0.08$ \\
\hline
\end{tabular}

\section{CONCLUSÕES E TRABALHOS FUTUROS}

No presente trabalho, o POMDP foi apresentado como uma solução para resolver o problema de planejamento e controle de um robô que se move por um sistema de estocagem de pilhas. $\mathrm{O}$ resultado da fase de planejamento e controle é a política, que determina as ações a serem tomadas pelo robô, a fim de maximizar a recompensa esperada. O POMDP é uma abordagem mais abrangente que o MDP, porém é mais difícil de se resolver.

Os experimentos mostram que o Algoritmo 1 apresentou sucesso na seleção das políticas de controle. Usando $T=1$, o algoritmo resolve o POMDP com baixo esforço computacional, possuindo complexidade $\mathcal{O}\left(|A||\Omega|\left|S^{2}\right|^{\}}\right.$em cada época de decisão. Algo razoável se compararmos o Algoritmo 1 com algoritmo de Sondik (também usado para resolver o POMDP), que possui ordem de complexidade $\mathcal{O}\left(|S||A|^{|\Omega|^{|T|}}\right)$.

Os resultados das funções valores obtidos na Seção 5 foram satisfatórios, uma vez que correspondem às ações que minimizam o critério de otimalidade escolhido (distância ou tempo) para recompensa $R\left(a_{k, i} \mid s_{k}\right)$. Na maioria das épocas de decisão há relativa discrepância entre o maior e o segundo maior valor de $V(b, a)$, possibilitando executar a ação $a_{i, j}$ (com maior $\left.V(b, a)\right)$ com grande margem de segurança. O Algoritmo 1 mostrou-se robusto apesar da pequena confiança na posição inicial. Se os valores da crença inicial forem mais consistentes, a diferença entre a maior e a segunda maior $V(b, a)$ é amplificada. Desta forma, o algoritmo se mostra adequado para fazer o rastreamento e planejamento das ações do robô até o alvo, desde que se tenha algum conhecimento (mesmo que mínimo) sobre a configuração inicial. Testes foram realizados utilizando uma distribuição uniforme para crença inicial, entretanto os resultados não foram razoáveis.

Há de se salientar, que os caminhos executados pelo robô foram ótimos, porém não são os únicos. Por exemplo, pode-se sair do estado $s_{9}$ em direção ao $s_{16}$ passando por $\left(s_{9}, s_{10}, s_{11}, s_{16}\right)$, ou $\left(s_{9}, s_{14}, s_{15}, s_{16}\right)$, no qual ambos são ótimos (em relação ao tempo e a distância). Como trabalhos futuros pretende-se estender a aplicação para um ambiente dinâmico, e que possua mais lugares críticos, como escadas e elevadores.

\section{REFERÊNCIAS}

Bellman, R. (1957). A markovian decision process. Journal of Mathematics and Mechanics, 6(5), 679-684.

Bonin-Font, F., Ortiz, A., and Oliver, G. (2008). Visual navigation for mobile robots: A survey. Journal of intelligent and robotic systems, 53(3), 263-296.

Chernov, V., Alander, J., and Bochko, V. (2015). Integerbased accurate conversion between $\mathrm{rgb}$ and hsv color spaces. Computers \& Electrical Engineering, 46, 328337.

Dudek, G. and Jenkin, M. (2010). Computational principles of mobile robotics. Cambridge university press.

Howard, R.A. (1960). Dynamic programming and markov processes.

Miehling, E., Rasouli, M., and Teneketzis, D. (2015). Optimal defense policies for partially observable spreading processes on bayesian attack graphs. In Proceedings of the Second ACM Workshop on Moving Target Defense, 67-76. ACM.

Nardi, L. and Stachniss, C. (2019). Uncertainty-aware path planning for navigation on road networks using augmented mdps. In Proc. of the IEEE Intl. Conf. on Robotics 8 Automation (ICRA).

Páll, E., Tamás, L., and Buşoniu, L. (2016). Analysis and a home assistance application of online aems 2 planning. In 2016 IEEE/RSJ International Conference on Intelligent Robots and Systems (IROS), 5013-5019. IEEE.

Pellegrini, J. and Wainer, J. (2007). Processos de decisão de markov: um tutorial. Revista de Informática Teórica e Aplicada, 14(2), 133-179.

Pineau, J., Gordon, G., and Thrun, S. (2004). Tractable planning under uncertainty: exploiting structure. Ph.D. thesis, Carnegie Mellon University, the Robotics Institute.

Spong, M.W., Hutchinson, S., Vidyasagar, M., et al. (2006). Robot modeling and control, volume 3. Wiley New York.

Thrun, S., Burgard, W., and Fox, D. (2005). Probabilistic robotics. MIT press.

Wang, C., Cheng, J., Wang, J., Li, X., and Meng, M.Q.H. (2018). Efficient object search with belief road map using mobile robot. IEEE Robotics and Automation Letters, 3(4), 3081-3088. 